\title{
Aggregation of Cyclodextrins: Fundamental Issues and Applications
}

\author{
Tânia F.G.G. Cova, Sandra M.A. Cruz, \\ Artur J.M. Valente, Paulo E. Abreu, \\ Jorge M.C. Marques and Alberto A.C.C. Pais \\ Additional information is available at the end of the chapter
}

http://dx.doi.org/10.5772/intechopen.73532

\begin{abstract}
The aggregation of cyclodextrins (CD) in aqueous solution is an old, yet still vastly unexplored topic that has been studied at least since the 1980s. At that time, few authors took into consideration the possibility of formation of aggregates for the interpretation of thermodynamic and thermophysical properties of CDs in aqueous solution. The aggregates appear at quite low CD concentrations and seem to encompass only a small number of CD molecules. They also occur in water in the presence of hydrophobic or amphiphilic moieties, including surfactants, assuming a preassembled state with the hydrophobic chains threading through one or two CDs. After a long period in which it has been neglected, CD aggregation is now a hot topic and one far from gathering consensus. In this chapter, a timely and critical review on the phenomenon of CD aggregation and the respective supramolecular properties, including some computational rationales, will be presented. A comprehensive summary of $\mathrm{CD}$ aggregates studied to date, indicating the formation conditions, characterization techniques, and applications, is also provided.
\end{abstract}

Keywords: cyclodextrins, self-aggregation, molecular dynamics simulations

\section{Introduction}

The aggregation of amphiphilic molecules in solution, including cyclodextrins (CD), can be considered as a microphase separation between polar and nonpolar phases. This involves both the hydrophobic and hydrophilic moieties of such molecules and induces the formation and growth of the so-called self-assembled structures. This is a spontaneous process in which the system components form ordered aggregates, typically involving an 
enthalpy gain in solvation, due to hydrogen bond formation, and possibly a gain in the entropy of bulk water (hydrophobic effect) [1-3]. The process is promoted by balanced attractive and repulsive interactions between molecules, or specific moieties, occurring from a less organized state (e.g., a solution or a disordered aggregate) to a final ordered state (e.g., a crystal). These soft interactions are generally weak, comparable to thermal energies, and noncovalent, with typical strengths varying from less than $5 \mathrm{~kJ} \mathrm{~mol}^{-1}$ for van der Waals forces to ca. $120 \mathrm{~kJ} \mathrm{~mol}^{-1}$ for hydrogen bonds [4]. Other relevant factors, such as the reversibility and flexibility, the interaction with the respective environment, and the mobility of the system components, determine the success of the molecular self-assembly process.

Despite a substantial amount of data is available on the thermodynamic aspects of assembly processes, the information on the $\mathrm{CD}$ aggregation behavior and detailed mechanisms of formation and dissociation is scarce. In particular, little is known about the cooperative nature of individual contributions of components for the CD aggregation/assembly process. This is clearly a topic requiring fundamental research, with direct implications for improving the supramolecular assembly design and fostering new formulation opportunities in this type of systems.

Table 1 summarizes some relevant physical-chemical parameters of the three most common natural cyclodextrins $\left(\alpha-, \beta-\right.$, and $\gamma_{-}$CDs). CDs are able to solubilize, protect, or transport a large variety of compounds, e.g., inorganic nonassociated salts $[5,6]$, surfactants [7-9], and nonpolar drugs, by forming inclusion and noninclusion complexes. These $\mathrm{CD}$-guest interactions are facilitated by the unique structure of $\mathrm{CD}$, which combines a hydrophilic outer surface with an internal lipophilic cavity. The extensive development of CD-based formulations has resulted in a strongly increasing amount of studies, in which the exceptional features of CDs have been described (see e.g., [10-16]). In the last decades, CDs have also been used for functionalization of organic [17] and inorganic $[18,19]$ materials and for the synthesis of nanosized and microsized aggregates [20-22]. This process is, in general, driven by hydrophobic interactions and is affected by a number

\begin{tabular}{llll}
\hline Property & $\alpha$-CD & $\beta$-CD & $\gamma$-CD \\
\hline $\mathrm{N}^{\circ}$ glucose unites & 6 & 7 & 8 \\
$\mathrm{MW}\left(\mathrm{g} \mathrm{mol}^{-1}\right)$ & 972 & 1135 & 1297 \\
Solubility in water $\left(\mathrm{g} \mathrm{L}^{-1}\right)$ & 145 & 18.5 & 232 \\
Outer diameter cavity, wide end $(\AA)$ & 13.7 & 15.3 & 16.9 \\
Inner diameter cavity, wide end $(\AA)$ & 5.7 & 7.8 & 9.5 \\
Volume cavity $\left(\AA^{3}\right)$ & 174 & 262 & 427 \\
No. water molecules ${ }^{*}$ inside cavity & 5.8 & 8.7 & 14.2 \\
\hline
\end{tabular}

*Assuming a volume of a water molecule equal to $30 \AA^{3}$.

Table 1. Some physical properties of $\alpha-, \beta$ - and $\gamma$-cyclodextrins [26, 33]. 
of factors such as the concentration of reactants, temperature, nature of solvent, and the addition of neutral or ionic co-solutes [23-25].

One of the most intriguing properties of CDs is the respective solubility. It should be noted that the solubility in water does not follow a common trend, with $\beta$-CD being much less soluble than the other two [26]. Such behavior has attracted researchers for several years, and different explanations have been proposed, based on the rigidity of the CD ring and intramolecular H-bonding or CD self-aggregation [27, 28]. The formation of aggregates can be enhanced upon formation of inclusion complexes, but in general, negligible amounts of aggregates (of ca. 200-300 nm in size) are formed in pure CD solutions. The extent of aggregation may also increase with increasing CD concentration [29].

A wide range of experimental data generated on $\mathrm{CD}$ aggregation have been reviewed (see e.g., Refs. $[2,3,30])$. However, the mechanisms of CD aggregation and CD complex aggregation have not yet been fully understood.

$\mathrm{CD}$ aggregation was originally recognized based on random observations and deviations from theoretical expectations. Several analytical techniques are now being optimized to specifically detect and characterize CD aggregates. In CD solutions, dynamic light scattering (DLS) [31, 32] and transmission electron microscopy (TEM) [32] have provided reliable evidence of the aggregation phenomenon. The latter has been recognized by several research groups that identified aggregates of different shapes, including spherical and elongated particles, welded fibers, and rods $[1,2,19,29]$.

A significant amount of microscopic data [1] and information from dynamic light scattering (DLS), nuclear magnetic resonance spectroscopy (NMR), and dialysis membrane permeation methods have been reported [1,3]. However, the full characterization of the CD aggregation behavior by these techniques has been hampered by the poor stability of the aggregates, which makes them sensitive to disruptive side effects [2, 32].

Computational methods, including molecular dynamics (MD) simulations, have been increasingly recognized as a valuable tool for the interpretation of experimental data [34-39]. The simulation of host-guest systems involving CD molecules and aggregates is already offering ways to explore a set of favorable events at different scales. The aggregation behavior and the interaction patterns of CDs, modified with both hydrophobic and hydrophilic groups, have been investigated by atomistic simulations in nonpolar solvents and in water $[34,35]$. Although most studies involving amphiphilic CDs have been conducted in aqueous solutions, the use of nonpolar solvents is also important in preparative and characterization procedures, as suggested in Ref. [34]. In the latter, dynamic light scattering observations demonstrated that CDs can form well-defined aggregates in nonpolar solvents (e.g., dichloromethane), displaying a hydrodynamic radius of ca. $80 \mathrm{~nm}$ and low polydispersity [34]. Electrostatic, dipolar, and dispersion forces have been recognized as playing a definite role in the formation and stabilization of these aggregates. The formation of even larger structures (such as micelles, vesicles, membranes, and nanoparticles) based on these smaller aggregates and their potential uses in pharmaceutical formulations have also been suggested. 


\section{General aspects}

\subsection{Controversial experimental evidence}

The aggregation of single CDs and CD-guest complexes, in water, has been described in a number of recent studies [2, 3, 13, 31, 37, 40,41]. Despite the efforts for understanding the factors that govern inclusion/binding with guest molecules, the precise manner in which CD molecules aggregate and the cooperative effects underlying this phenomenon are much less studied and still far from consensual.

The classical assumption states that inclusion complexes between CDs and guest molecules are always formed in "ideal" solutions, with individual complexes, independent of each other. However, the treatment of the interaction between system components is oversimplified, since CDs can form both inclusion and noninclusion complexes and water-soluble aggregates. In some cases, the aggregation results in the opalescence of aqueous CD solutions. However, the reduced diameter of the aggregates is smaller than the wavelength of visible light, and more often, the formation of clear solutions is promoted.

The first reference to the occurrence of self-aggregation dates back to 1983, when Koichiro and co-workers [42], based on viscosity and activity coefficient data of aqueous solutions of $\alpha$ and $\gamma$-cyclodextrins, suggested the occurrence of dimers or larger aggregates. These authors have also pointed out that such aggregates are acting as "structure making" [43] by tightening water-water hydrogen bonding. Ten years later, Häusler and Müller-Goymann [44] have observed that at concentrations above $50 \%(\mathrm{w} / \mathrm{w})$, hydroxypropyl (HP)- $\beta$-CD self-aggregates, leading to an increase in the solution viscosity. They also found that the addition of chaotropic solutes (e.g., urea and $\mathrm{NaCl}$ ) tends to decrease the viscosity of solutions as a consequence of CD disaggregation. A similar observation was found for $\gamma$-CD $(10 \% \mathrm{~m} / \mathrm{v})$ solutions [45], at physiological $\mathrm{pH}$. In both works, the self-aggregation of $\mathrm{CD}$ monomers is justified by intermolecular H-bonding interactions promoted by the hydrophilic rims [44, 45].

Coleman et al. [46] extended their studies to $\beta-C D$, the less soluble of all native CDs, and found that the addition of water structure-breaking solutes or an increase of the solution $\mathrm{pH}$ (at values higher than 12.5) in order to ionize the - $\mathrm{OH}$ groups leads to an increase in solubility. This evidence led them to hypothesize that the solubility of CDs is related with interactions between $\mathrm{CD}$ aggregates and the water through the formation of two chains of hydrated $\beta$-CD molecules forming a rod-like aggregate. These authors also argue that the structure of water has an important role on the aggregation/disaggregation of CDs.

The quantification of size and mass percentage of aggregates was only made possible, in a systematic manner, during the last decade. It has been concluded, by using photon correlation spectroscopy (PCS), that in a $12 \mathrm{mM} \mathrm{CD}$ aqueous solution, large polydisperse aggregates of 200-300 nm in size were formed [29]; however, the respective mass percentage is quite small when compared to that of free CD. For example, the mass contribution of $\alpha$-CD aggregates is ca. $0.8 \%(0.096 \mathrm{mM})$ assuming coils or $0.001 \%(0.12 \mu \mathrm{M})$ considering spheres [29]. For other CDs, the mass contribution of aggregates is also residual, i.e., $0.0011 \%(0.13 \mu \mathrm{M})$ for $\beta$-CD [47] and $0.02 \%(0.154 \mathrm{mM})$ for $\gamma$-CD solutions [45]. He et al. [48] found a bimodal distribution in dynamic 
light scattering (DLS) data of $\alpha-, \beta-$, and $\gamma$-CD aqueous solutions, with mean hydrodynamic radii of less than $1 \mathrm{~nm}$ and higher than $60 \mathrm{~nm}$ for the fast and slow components (attributed to monomer and aggregated CDs, respectively). This has been revisited by Puskás et al. [49] by using the same technique. However, contrarily to previous work, only $\gamma-\mathrm{CD}$ aggregates have been confirmed. In other studies [50,51], the formation of CD aggregates of globular shape, at $[\beta-C D]=3 \mathrm{mM}$, with a minimum hydration radius of ca. $90 \mathrm{~nm}$, was reported, based on data from different techniques, including DLS, cryo-TEM, and electron spin resonance (ESR) probe spectroscopy. However, at higher CD concentrations, these particles coexist with other structures as large as a few micrometers. These aggregates tend to increase with CD concentration, suggesting cooperative aggregate-aggregate interactions. In addition, Rao and Geckeler [52] have concluded that the formation of $\beta$-CD supramolecules can also occur in aqueous solutions at room temperature. By increasing the stirring time and the concentration from 4 to $10 \mathrm{mM}$, it was possible to follow the formation of cage-like structures (with an average size of $7.5 \mathrm{~nm}$ ), after $5 \mathrm{~h}$ stirring, evolving to channeled structures (with $260 \mathrm{~nm}$ length) after $72 \mathrm{~h}$ stirring.

The thermodynamic parameters of $\beta$-CD self-assembly have been studied, assuming that CDs behave as colloids [36]. For that, the scattering intensity of a set of $\beta$-CD solutions of different concentrations has been measured; by plotting the scattering intensity as a function of $\beta$-CD concentration, two different regimes were observed below and above the critical aggregation concentration $(\mathrm{cac}=1.6 \mathrm{mM})$ : at $\mathrm{c}<\mathrm{cac}$, and only monomers (the occurrence of small aggregates cannot be also neglected) exist in solution. However, at $\mathrm{c}>\mathrm{cac}$, the scattering intensity increases linearly with $\beta$-CD concentration, indicating the presence of two different set of aggregates of $60.0 \mathrm{~nm}$ and $120 \mathrm{~nm}$ in size. Using the pseudo-phase model, the free energy of aggregation was calculated to be $-15.95 \mathrm{~kJ} \mathrm{~mol}^{-1}$ at $298.15 \mathrm{~K}$. From the dependence of the cac value on the temperature, the variation of the aggregation enthalpy and, consequently, the aggregation entropy (at $298.15 \mathrm{~K}$ ) were estimated to be $-26.48 \mathrm{~kJ} \mathrm{~mol}^{-1}$ and $-35.32 \mathrm{~J} \mathrm{~K}^{-1} \mathrm{~mol}^{-1}$, respectively. These values for thermodynamic parameters suggest that (a) aggregation is enthalpy driven, and (b) aggregates are formed by noncovalent interactions [53]. Following the same approach, the cac values of $\alpha-, \beta-$, and $\gamma$-CDs were also measured by using a permeability technique, based on dialysis membranes with molecular weight cut off higher than $2 \mathrm{kDa}[3,54]$. Inspecting the flux of CDs as a function of concentration, two different regimes have been found which were attributed to the formation of aggregates. The calculated values for the cac of $\alpha-, \beta-$, and $\gamma$-CDs are 12.2, 6.1, and $7.2 \mathrm{mM}$, respectively.

Recently, a thermodynamic study [55] using isodesmic and K2-K self-assembly models was performed on $C D$ derivatives (HP- $\beta-C D, H P-\gamma-C D$, and sulfobutylether(SBE)- $\beta-C D$ ). The isodesmic model assumes that the Gibbs energy and equilibrium constant $(\mathrm{K})$ are equal in each addition of a monomer to an aggregate (for details see Ref. [55]). The K2-K model is a modified version of the former, in which the $\mathrm{K}$ value of the first step of the self-assembly is different from those of the remaining steps. The respective cac values and aggregate sizes were also determined, using DLS and ${ }^{1} \mathrm{H}$ NMR and TEM, respectively. These CDs displayed similar cac values of ca. $2 \%(\mathrm{~m} / \mathrm{v})$. Three different groups of particle sizes were also identified based on correlation functions: (i) a group with ca. $1 \mathrm{~nm}$ corresponds to a single CD molecule, (ii) a group with size values ranging from ca. $30 \mathrm{~nm}$ to $60 \mathrm{~nm}$ for HP- $\gamma-C D$ and from ca. 10-70 nm for SBE- $\beta$-CD and from ca. $100 \mathrm{~nm}$ to $200 \mathrm{~nm}$ for $\mathrm{HP}-\beta-\mathrm{CD}$, and (iii) a small group with larger 
aggregates attaining $1 \mu \mathrm{m}$, for $\beta$-CD derivatives, and from ca. $140 \mathrm{~nm}$ to $1 \mu \mathrm{m}$ for HP- $\gamma$-CD. It was shown that aggregation results from some cooperative contributions, with the first step of the aggregation being less favorable than the subsequent ones. A thermodynamic analysis indicated that the aggregation process was spontaneous, exothermic, and associated to an entropy loss. The calculated standard free energies range from $-7.1 \mathrm{~kJ} \mathrm{~mol}^{-1}$ for SBE- $\beta$-CD to -10.6 for HP- $\gamma-C D$, and the enthalpy values were $-20.6,-27.5$, and $-46.3 \mathrm{~kJ} \mathrm{~mol}^{-1}$ for $\mathrm{HP}-\beta-\mathrm{CD}$, SBE- $\beta-C D$, and HP- $\gamma-C D$, respectively [55].

The dynamics of CDs in aqueous solution has been fully assessed using different NMR techniques by Valente et al. [56, 57]. The analysis of ${ }^{1} \mathrm{H}$ NMR self-diffusion of deuterium solutions of CDs shows that, for all three natural CDs, diffusion coefficients depend linearly on the CD volume fraction, suggesting that these molecules are behaving as nonaggregate hard spheres. A similar conclusion was reached from the analysis of the dependence of mutual diffusion coefficients on CDs concentration [58-60].

A more sensitive parameter, related to the volume of the diffusing particle, is the transverse magnetization relaxation time $T_{2}$ or the spin-spin relaxation rate $R_{2}\left(=1 / T_{2}\right)$. In a perfectly homogeneous magnetic field, the $R_{2}$ relaxation rate can be measured directly from the free induction decay in the time domain or the full width at half height of the resonance in the frequency domain. The dependence of $R_{2}$ as a function of CD volume showed that no aggregation occurs. However, the presence of more transient aggregates cannot be excluded for cases in which the lifetime of the aggregate is short compared to the respective tumbling time. The presence of very large aggregates, not visible in the NMR spectra on account of their slow rotational tumbling, cannot also be ruled out. A different but complementary aspect is related to the mechanism of interaction. For further insight, the aggregation of $\alpha-, \beta-$, and $\gamma_{-} \mathrm{CDs}$ in aqueous solutions was addressed by focusing on the CD-CD interactions using deuterium relaxation rates $\left(R_{1}\right)$ for deuterium-labeled CDs. In this particular case, the dependence of $T_{1}\left(=1 / R_{1}\right)$ on the CD concentration, for all CDs, was explained by the equilibrium between monomeric and dimeric CDs and, again, no evidence in favor of large aggregates of CDs involving a nonnegligible fraction was found [57].

The formation of aggregates in aqueous solutions containing CDs can be promoted by the presence of guest compounds, which upon inclusion can also contribute to understand and predict the $C D$ aggregation behavior. The structure and nature of the guest molecule can thus affect the CD aggregation process. CD-guest complexes are, most often, simply formed by one guest molecule and one CD molecule. However, ternary complexes are also frequently described [61], where water-soluble polymers [62, 63], metal ions, or organic salts [64] are used to potentiate some CD effect.

The coexistence of inclusion and noninclusion complexes in aqueous solutions containing CDs has been documented and associated with the formation of aggregates based on these complexes. The first evidence for aggregation with complexes involving CDs and lipophilic guests was reported by Mele and co-workers [65] in 1998. Later studies [32], with contradictory or ambiguous results, fostered further investigation on this phenomenon.

The formation of CD-guest-based aggregates in the nanosize range has been confirmed by DLS and TEM analyses and associated to a negative deviation from linearity. This 
type of aggregation occurred for a CD concentration of ca. 10\% (wt/vol) [32]. The selfassociation of $\mathrm{CD}$ complexes can explain the observed decrease in the activity coefficient with increasing $\mathrm{CD}$ concentration and the dependence of the complex stoichiometry on the method applied.

Several model compounds [32] have been recently used to investigate the effect of the physicochemical properties of the guest molecules on the CD aggregation behavior. For instance, the impact of a set of esters of para-hydroxybenzoic acid, differing in the side chain length, on HP- $\beta-C D$ aggregation was recently evaluated resorting to permeation experiments, DLS and MS. The number and size of CD aggregates $(<200 \mathrm{~nm})$ increased in the presence of longer guests. However, no clear relation was found between the extent of aggregate formation and the CD concentration.

The premicellar association of inclusion complexes of cationic surfactants and $\beta-\mathrm{CD}$ followed by micellar association of the inclusion complexes has also been suggested based on NMR studies. In addition, micelle-like assemblies with diameters exceeding $200 \mathrm{~nm}$ have been observed in aqueous solutions containing trans- $\beta$-carotene and $\beta-\mathrm{CD}$ and $\gamma-\mathrm{CD}$ [2].

The structure and size of these CD aggregates are clearly affected by water molecules and hydration shells [2]. Although the ability of CDs to self-assemble to form aggregates is well documented, it has also been shown that the aggregates are very unstable. Attempts to stabilize nanosize self-assembled CD aggregates of the native CDs, and their hydrophilic and monomeric derivatives have not been successful so far.

\subsection{Common arrangements in $\mathrm{CD}$ aggregates}

There are two typical crystal structures for native CDs: cages and channels (see Figure 1) $[22,66]$. The cage arrangement occurs when CDs are grouped crosswise, displaying a herringbone pattern (Figure 1a), or are aligned in adjacent layers leading to a brick-like pattern (Figure 1b). In both cases, the formation of inclusion complexes is prevented, as the CD cavities are blocked on both portals by neighboring CDs.

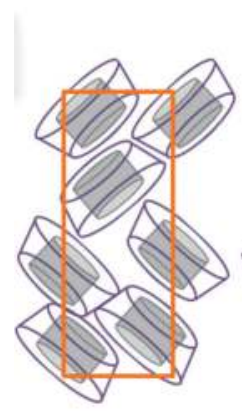

(a)

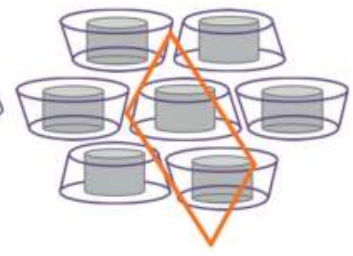

(b)

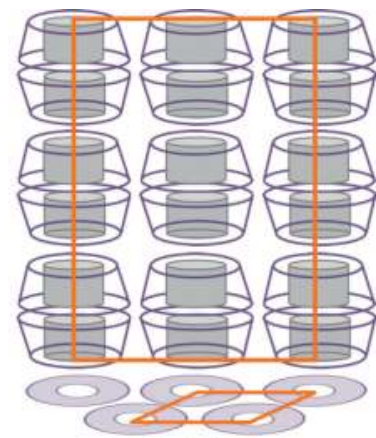

(c)

Figure 1. Schematic representation of packing structures of (a) cage-type; (b) layer-type CD; and (c) head-to-tail channeltype CD crystals. Adapted from Ref. [66]. 
The channel-type assembly is observed if the CDs are stacked in columns so that cavities are aligned to produce channels (Figure 1c). While the channel arrangement can be converted into a cage structure by water sorption/desorption cycles, with an intermediated amorphous state, the latter usually results from rapid recrystallization of CDs. After reaching sorption equilibrium, $\mathrm{CD}$ molecules undergo a slow rearrangement to the cage structure with defined water content [66]. Similar patterns are generally found in the crystal structures of CD inclusion complexes [66]. When the entire guest molecule is small enough to be included inside a single CD cavity, cage-type structures are formed. On the other hand, a channel-type structure is observed in the presence of long-chain molecules as guests (e.g., polymers). Rusa and co-workers [2, 67] have reported the encapsulation of poly( $\varepsilon$-caprolactone) and poly(L-lactic acid) into $\alpha$ - and $\gamma$-CDs. The inclusion of a polymeric structure inside the CD cavity induces the formation of channel-like structures, being those with $\alpha$-CD more stable due to the increase of hydrophobic $\mathrm{CD}$-polymer interactions. By using an appropriate experimental procedure, the authors were able to produce a solid-state channel packing of CDs containing only water molecules inside the cavities [68]. The same group has also observed the inclusion of poly(vinyl alcohol) (PVA) into CD by taking advantage of the freezing-thawing process for PVA gelation [69]. In this process, the gelation of PVA-containing composites occurs, taking into account two different types of cross-linking: (i) the hydrogel-bonding naturally observed during the freezing-thawing process and (ii) the CD-CD aggregation resulting from channel-type arrangements [69].

\subsection{Applications of CD aggregates}

As already mentioned in previous sections, a variety of CD-based aggregates can be formed under different conditions (e.g., concentration, solvent medium, and temperature). These include native and modified CDs, inclusion complexes and the respective aggregates and also rotaxanes and polyrotaxanes, nanotubes, and other high-order structures, such as nanospheres and network aggregates [70]. The potential uses of these self-assembled nanomaterials have been explored for advanced applications, ranging from drug solubilization and drug delivery [71], selective binding [72], and controlled adsorption [72]. In pharmaceutical and biomedical fields, it is expected that such applications may include (i) the nanoencapsulation of drugs in the hydrophobic interchain volumes and nanocavities of modified CDs, which can be used as drug carriers or pharmaceutical excipients, (ii) anticancer phototherapy, (iii) gene delivery, and (iv) protection of unstable active components through the formation of inclusion complexes [70]. Several interesting examples of these potential applications have been focused on amphiphilic CDs, which allow to easily modulate both hydrophobic/hydrophilic and self-assembly properties, by grafting different substituents on the portals of native $\mathrm{CDs}[70,73]$. For instance, supramolecular assemblies based on CD/porphyrin nanoassemblies have been studied in vitro [74] as potential nanotherapeutics in A375 human melanoma cells. Other micellar structures and spherical vesicles based on CD-perylene conjugates have been designed to be included in fluorescence sensory and photoresponsive materials, photoinduced electron transfer systems, and organic electronic devices [75]. The self-aggregation of amphiphilic CDs has also been explored for drug delivery applications, as they are able to capture selectively drug molecules, displaying enhanced solubilization capacity [71]. The affinity of amphiphilic CDs for incorporation in model and biological membranes has 
also been investigated and explored for preparation of functionalized lipid membranes and improved biomimetic systems [73, 76]. A broader range of potential applications of CD aggregates are compiled in recent publications such as Refs. [70, 73] and references therein.

\section{Computational observations}

Irrespective of the disparate observations, the self-aggregation capability must be affected by the type of CD present in the aqueous solution. While the aggregates of $\alpha-C D$ and $\gamma-C D$ can be completely removed by standard filtering procedures, the formation of $\beta$-CD aggregates (at least as dimers or possibly larger aggregates) persists in solution, displaying fast aggregation kinetics. This suggests that the hydrophilic CD portals play a definite role in the aggregation process [29]. The disruption of hydrogen bond networks by ionization (or functionalization) increases solubility and may suppress aggregation.

The presence of aggregates in solutions containing structure-breaking solutes, in which the solubility of $\beta-C D$ is enhanced, has provided new insights into this unusual behavior. The low solubility of $\beta$-CD (see Table 1 ) has been explained by the presence of aggregates and the respective unfavorable interaction with the hydrogen bond network of bulk water [46]. Note that aspects pertaining to the binding energy in the solid state cannot be disregarded. The relevance of understanding the mechanistic details of the CD aggregation phenomena encompasses either controlling/preventing the formation of aggregates (that preclude the development of specific formulations and the product development) or designing novel formulation strategies.

Computer simulations have been used to rationalize the experimental findings concerning recognition [77], inclusion [36, 77-79], and aggregation [34, 35, 37, 39, 80]. The cooperative binding of at least two CD monomers to a guest molecule has been considered the driving force responsible for self-assembly processes in the construction of CD-based nanoarchitectures [78]. For aggregation processes without using guests, the assembly is usually driven by the hydrophilic portals (in native CDs) and by interactions between substituent chains of CD derivatives with the neighboring cavities of other CDs [78].

The orientational patterns of inter-glucopyranose hydrogen bonds at the secondary portal of $\beta-C D$ and the respective effect on the CD structure and dimer binding/stability in polar and nonpolar solvents have been explored by van der Spoel et al. [77] in the presence of various guest molecules. It was demonstrated, based on MD simulations and free energy calculations, that polar solvents with stronger hydrogen bond accepting abilities can easily disrupt intermolecular hydrogen bonds, resulting in less stable dimers. Also, the guest models included in the channel-type cavity increase the binding affinity between CD monomers, particularly in polar solvents [77]. Using a similar computational approach, the authors have explored the effect of three different dimerization modes of $\beta$-CD molecules and the presence of isoflavone drug analogues in the construction of CD-based nanostructured materials. It was demonstrated that the cooperative binding of $\mathrm{CD}$ cavities to guest molecules favors the dimerization process and, consequently, the overall stability and assembly of the CD nanostructures. It 
was also proved that the desolvation of CD dimers and entropy changes upon complexation cooperatively contributes to the binding process [78].

Another study [37] focused on the spontaneous adsorption of native CDs and the respective aggregates and the related dependence on temperature. It was found that the adsorption of both individual CDs and small CD aggregates (ca. 20 molecules) to the solution/air interface is negligible. The solute-solute interactions were significantly larger for $\beta-\mathrm{CD}$ than for $\alpha-\mathrm{CD}$ at $298 \mathrm{~K}$, and the dependence of these interactions on temperature was more relevant for the smaller CD, which displayed a more favorable aggregation at $283 \mathrm{~K}$ than at $298 \mathrm{~K}$. The dynamic exchange of hydrogen bonds between the CD hydroxyl groups and the neighboring water molecules indicated a much larger occupancy for individual intramolecular H-bonds in $\beta$-CD.

In what follows, the CD-CD interactions [57], for deuterium labeled CDs, in aqueous solutions are further explored by atomistic simulations. Two types of systems are defined, one in which the $\beta-C D$ is free in water and three others in which two $\beta$-CDs are present and may form dimers. In what concerns the latter, these include initial arrangements with proximity of one primary portal and one secondary portal (PS), two primary portals (PP), and two secondary portals (SS), as shown in Figure 2.

The molecular dynamics simulations were performed with Gromacs (version 4.6.5), using the all-atom amber99sb [81] force field and the TIP3P water model. The initial coordinates of the $\beta$-CD were extracted from the RCSB protein data bank (PDB code: 1DMB), and partial charges were generated using the R.E.D.D. Server [82].

In each system, the molecules were accommodated in a cubic box (7.5 nm edge-length) containing approximately 13,000 explicit TIP3P water molecules. To obtain a starting configuration, each system was firstly subjected to an energy minimization step. All the calculations were carried out in NPT ensemble with periodic boundary conditions at a constant temperature of $300 \mathrm{~K}$ and a pressure coupling of 1.0 bar, respectively, to V-rescale and Berendsen external baths. A standard time step of $2 \mathrm{fs}$ was used for both equilibration and production runs. A cut-off of $0.9 \mathrm{~nm}$ was used for calculating the Lennard-Jones interactions. Electrostatic interactions were evaluated using the particle mesh Ewald method [83]. Constraints were applied for bond lengths with the LINCS algorithm [84].

Equilibrium properties, structure, and dynamics of $\beta$-CD systems were calculated for the simulation runs of $50 \mathrm{~ns}$ after the systems were equilibrated for 2 ns. Geometric clustering was performed to identify dominant CD-CD structures, sampled during the MD simulations. The algorithm for cluster analysis is based on the hierarchical (top-down) approach [85] and

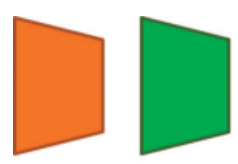

PS

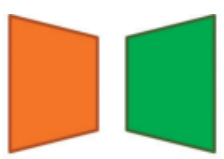

PP

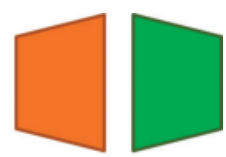

SS

Figure 2. Schematic illustration of the pairwise initial arrangements of $\beta$-CDs, with facing primary and secondary portals (PS), two primary portals (PP), and two secondary portals (SS), with the colors used to distinguish $\beta$-CD molecules. 
allows evaluating the conformational prevalence of each CD-CD structure, by determining dominant clusters based on the root mean square deviation of the atom positions between all pairs of structures. For each CD-CD arrangement, the number of neighboring structures is calculated for RMSD values of $0.35 \mathrm{~nm}$. Figure 3 presents the behavior of CD-CD structures in different simulation runs, each corresponding to a different initial arrangement. For each $\mathrm{CD}$ backbone, the center of mass (COM) of the oxygen atoms at the secondary portal (S) was defined as the reference point for evaluating aggregated and nonaggregated structures and the possible rotation or tilt of the CD molecules.

For PS and PP as initial arrangements, a significant evolution is observed in the relative positioning of the two molecules. The PS and PP initial arrangements display an almost complete rotation or a tilt of one molecule with respect to the other, leading to most favorable SS and PS arrangements, respectively. The "intermediate" PS arrangement increases the CD-CD interactions through partial inclusion of some P groups in the hydrophobic cavity of the other CD molecule. The initial SS arrangement prevails over the course of the simulation with a typical COM distance of $0.46 \mathrm{~nm}$. In addition to intramolecular hydrogen bonds, the two CD molecules can form additional intermolecular hydrogen bonds, optimizing the CD-CD interaction. PS is an intermediate arrangement between the most (SS) and the least stable (PP) arrangements. The PP arrangement of the CD pair involves weaker interactions between $\mathrm{P}$ groups of the two molecules, producing a low prevalence, relatively open aggregate (COM distance of $0.8 \mathrm{~nm}$ ), suggesting a relatively poor clustering of this dimeric aggregate.

The rotational autocorrelation functions (ACF) corresponding to the motion of each $\mathrm{CD}$, free or mostly in dimer arrangement, were also inspected. Two alternatives were tested for the

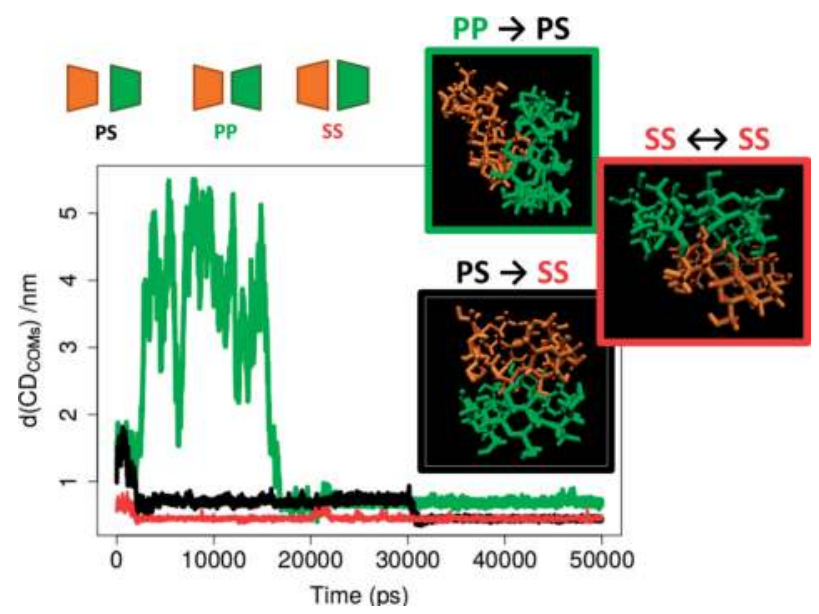

Figure 3. Distribution of distances between the centers of mass of $\beta-C D$ molecules, defined by the oxygen atoms of the secondary portals. Right panels illustrate the final conformations for the imposed PS, PP, and SS initial arrangements of $\beta$-CD molecules, in aqueous solution, sampled during the MD simulations at $300 \mathrm{~K}$ and identified by geometric cluster analysis. The color codes for CD molecules are as in Figure 1, while the initial arrangements are represented in black, green, and red, for PS, PP, and SS, respectively. 
definition of the molecule fixed rotating vector defined by the C2-D (see Figure 4) bond (vector) and resulting from the use of three atoms (triplet), those of the C2-D bond and the adjacent carbon C3, and defined as the cross product of vectors C3-C2 and C2-D.

The ACF curves are similar for these two cases and are represented in Figure 5. The curves were fitted from 0 up to 500.0 ps to a one-parameter exponential and reflect the slower decay for the dimer situation, $\tau_{0}=1250 \mathrm{ps}$, much larger than for the free CD, $\tau_{0}=448 \mathrm{ps}$. These values are of order of magnitude of those experimentally obtained.

The organization of CD molecules in aggregates when in the presence of guest-entities clearly deserves further attention. As an example of the behavior found for more complex systems, simulations were performed to study the inclusion complex of $\beta-\mathrm{CD}$ and poly(vinyl alcohol) (PVA) molecules in water. The importance of this polymer is related to the ability to form hydrogels exhibiting a high degree of swelling in water that has demonstrated a great potential to act as a matrix for many applications, including drug delivery [86], wound dressing [87], and sensors [88]. More recently, it has been found that such broad applications of PVA result from its ability to behave as an amphiphilic polymer [89]. This latter feature is relevant for studying the ability of CDs for forming host-guest or aggregate complexes. In this example, three molecular dynamics simulations were performed in systems containing two $\beta-C D$

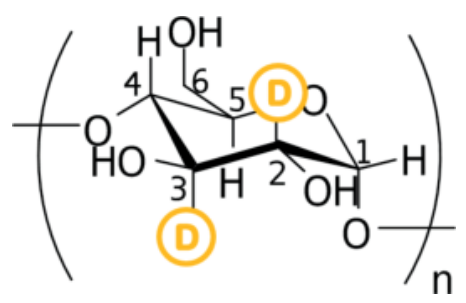

Figure 4. Cyclodextrin structures $(\alpha-C D, n=6 ; \beta-C D, n=7$; and $\gamma-C D, n=8)$.

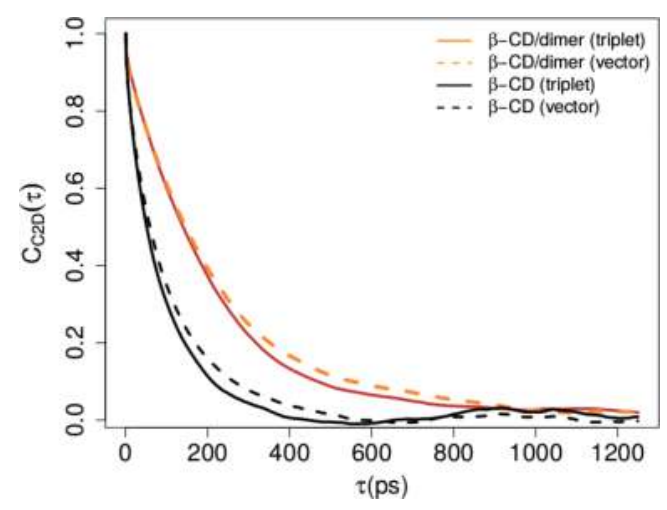

Figure 5. Average autocorrelation function of C2-D bond for CD molecules in the monomeric and dimeric states, in water at $300 \mathrm{~K}$ (black and orange curves, respectively). 
(denoted as A and B) molecules with 1 PVA, 2 PVA (A and B), and 10 PVA (A to J) oligomers, respectively. The main results are illustrated in Figure 6.

CDs are able to form aggregates at early stages of the simulations, and PVA seems to promote the formation of $\mathrm{CD}$ dimers $\left(\mathrm{Dm}_{\mathrm{AB}}\right)$. Indeed, PVA contains both hydrophilic and hydrophobic groups that may interact either with the outside part of the CDs or form inclusion complexes; the latter is shown by the snapshot in Figure 6(b), while the former appears for simulations of
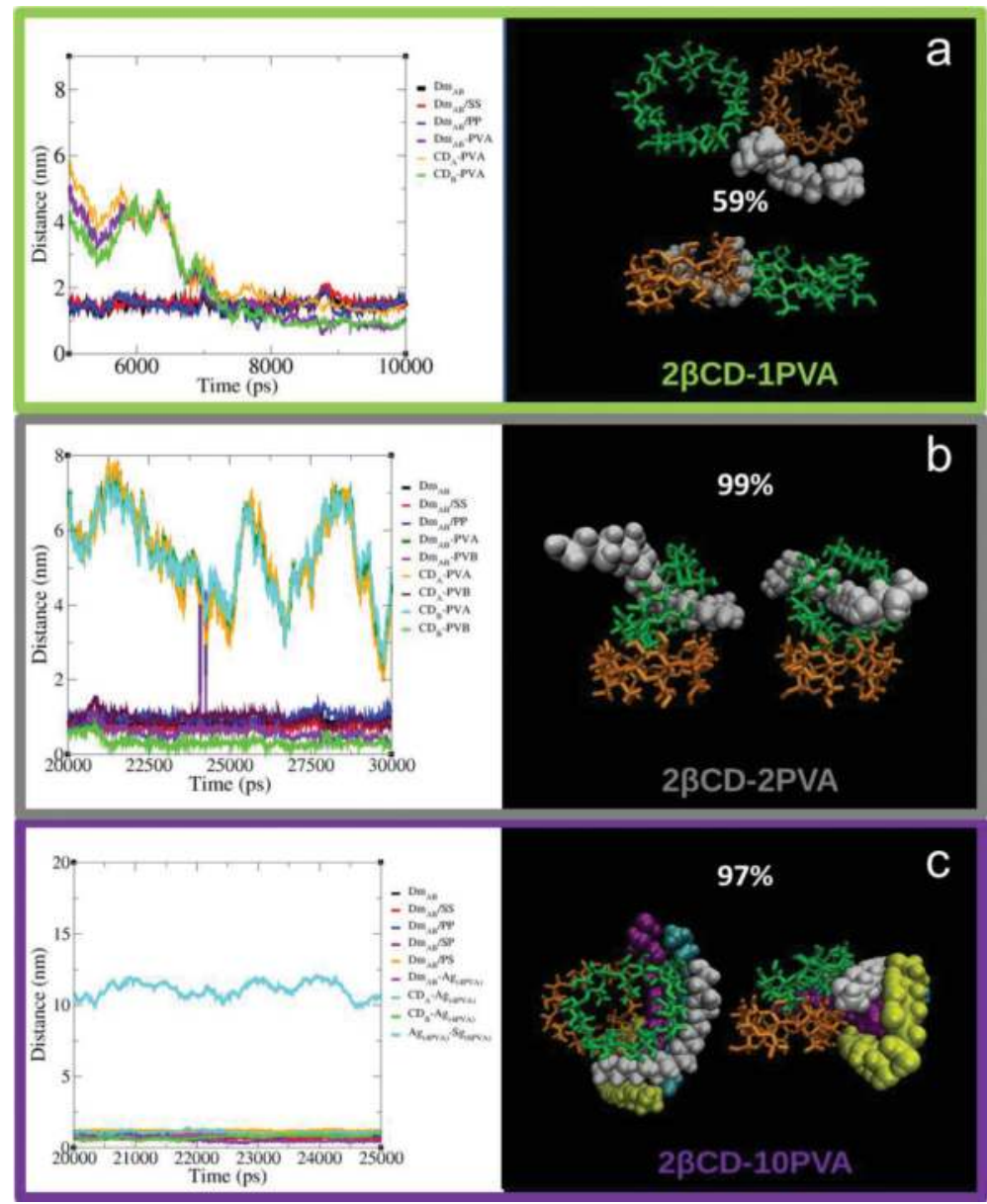

Figure 6. Summary of the MD simulations between $\beta-C D$ and PVA molecules. Left panels present the measured distances between the center of mass of the groups formed by (i) each $\beta-C D$ in the dimer $\left(\mathrm{Dm}_{\mathrm{AB}}\right)$, (ii) the secondary-secondary portals (SS), the primary-primary portals $\left(\mathrm{Dm}_{\mathrm{AB}} / \mathrm{PP}\right)$, the secondary-primary portals (PS), $\mathrm{Dm}_{\mathrm{AB}}-\mathrm{PVA}, \mathrm{Dm}_{\mathrm{AB}}-\mathrm{PVB}, \mathrm{CD}_{\mathrm{A}}-$ PVA, CD $-\mathrm{AVB}, \mathrm{CD}_{\mathrm{B}}-\mathrm{PVA}, \mathrm{CD}_{\mathrm{B}}-\mathrm{PVB}, \mathrm{Dm}_{\mathrm{AB}}-\mathrm{PVA}$ aggregate $\left(\mathrm{Ag}_{(4 \mathrm{PVA})}\right), \mathrm{CD}_{\mathrm{A}}-\mathrm{Ag}_{(4 \mathrm{PVA})^{\prime}} \mathrm{CD}_{\mathrm{B}}-\mathrm{Ag}_{(4 \mathrm{PVA})^{\prime}}$ and $\mathrm{Ag}_{(4 \mathrm{PVA})}-6$ separated PVA molecules $\left(\mathrm{Sg}_{(6 \mathrm{PVA})}\right)$. Right panels illustrate the 3 dominant conformations (accounting for more than $50 \%$, panel a, and $90 \%$, panels b and c, of the occurrences) sampled during the equilibrated parts of the production runs, at $300 \mathrm{~K}$, in aqueous solution. The force field F-85 [90] was used for the CDs. The electrostatic charges of PVA were calculated using the R.E.D. server and the remaining terms generated by ACPYPE [91]. 
2 CDs with 1 PVA (Figure 6(a)) and 2 CDs with 10 PVAs (Figure 6(c)). It is also noted that the prevalence of the type of configurations in Figure 6(b) and Figure 6(c) across the simulation is very high (above 95\%), which is a strong indication that such complexes are stable; for 2 CDs with 1 PVA, even though the prevalence of the typical configuration represented in Figure 6(a) is above $50 \%$, it is much less than the other two cases. Nonetheless, it is apparent from the distance versus time plot in Figure 6(b) that the PVA is able to exit the CD pocket in some instances and, then, reform the inclusion complex. In turn, it is particularly interesting to notice from the simulation of 2 CDs with 10 PVAs that the concomitant aggregation of the CDs with various molecules of PVA appears to be relatively stable complex and likely to delay or even prevent the formation of inclusion complexes (not observed during the course of the simulation).

\section{Concluding remarks}

CD aggregation is still a fertile ground for research, both from the experimental and computational points of view. It was seen that conflicting evidences are provided experimentally, calling for a comprehensive explanation of the involved phenomena, and that detailed, interaction-based, mechanistic rationales from computation are still much needed. It is expected that, in the next years, this subject will benefit from substantial efforts on both grounds.

\section{Acknowledgements}

The Coimbra Chemistry Centre is supported by the Fundação para a Ciência e a Tecnologia (FCT), Portuguese Agency for Scientific Research, through the Projects PEst-OE/QUI/ UI0313/2014 and POCI-01-0145-FEDER-007630. Sandra M.A. Cruz thanks the postdoc grant from Coimbra Chemistry Centre (Contract DPDRH-56-15-401). Tânia F.G.G. Cova also acknowledge the PhD research Grant SFRH/BD/95459/2013 assigned by FCT.

\section{Author details}

Tânia F.G.G. Cova, Sandra M.A. Cruz, Artur J.M. Valente*, Paulo E. Abreu, Jorge M.C. Marques and Alberto A.C.C. Pais

*Address all correspondence to: avalente@ci.uc.pt

CQC, Department of Chemistry, University of Coimbra, Coimbra, Portugal

\section{References}

[1] Kurkov SV, Loftsson T. Cyclodextrins. International Journal of Pharmaceutics. 2013;453: 167-180 
[2] Ryzhakov A, Do Thi T, Stappaerts J, Bertoletti L, Kimpe K, Sá Couto AR, Saokham P, Van den Mooter G, Augustijns P, Somsen GW, Kurkov S, Inghelbrecht S, Arien A, Jimidar MI, Schrijnemakers K, Loftsson T. Self-assembly of cyclodextrins and their complexes in aqueous solutions. Journal of Pharmaceutical Sciences. 2016;105:2556-2569

[3] Saokham P, Sá Couto A, Ryzhakov A, Loftsson T. The self-assemble of natural cyclodextrins in aqueous solutions: Application of miniature permeation studies for critical aggregation concentration (cac) determinations. International Journal of Pharmaceutics. 2016;505:187-193

[4] Whitesides GM, Boncheva M. Beyond molecules: Self-assembly of mesoscopic and macroscopic components. Proceedings of the National Academy of Sciences. 2002;99:4769-4774

[5] Assaf KI, Ural MS, Pan F, Georgiev T, Simova S, Rissanen K, Gabel D, Nau WM. Water structure recovery in chaotropic anion recognition: High-affinity binding of dodecaborate clusters to $\gamma$-cyclodextrin. Angewandte Chemie International Edition. 2015;54:6852-6856

[6] Terekhova I, Chibunova E, Kumeev R, Kruchinin S, Fedotova M, Kozbiał M, WszelakaRylik M, Gierycz P. Specific and nonspecific effects of biologically active inorganic salts on inclusion complex formation of cyclodextrins with aromatic carboxylic acids. Chemical Engineering Science. 2015;122:97-103

[7] Valente AJM, Söderman O. The formation of host-guest complexes between surfactants and cyclodextrins. Advances in Colloid and Interface Science. 2014;205:156-176

[8] Martín VI, Ostos FJ, Angulo M, Márquez AM, López-Cornejo P, López-López M, Carmona AT, Moyá ML. Host-guest interactions between cyclodextrins and surfactants with functional groups at the end of the hydrophobic tail. Journal of Colloid and Interface Science. 2017;491:336-348

[9] Shang C, Chen Y, Zhang C, Deng Z, Wu Z. Destruction of chitosan capsules based on host-guest interaction and controllable release of encapsulated dyes. Journal of Applied Polymer Science. 2017;134:45229

[10] Venuti V, Rossi B, Mele A, Melone L, Punta C, Majolino D, Masciovecchio C, Caldera F, Trotta F. Tuning structural parameters for the optimization of drug delivery performance of cyclodextrin-based nanosponges. Expert Opinion on Drug Delivery. 2017;14:331-340

[11] Liao R, Lv P, Wang Q, Zheng J, Feng B, Yang B. Cyclodextrin-based biological stimuli-responsive carriers for smart and precision medicine. Biomaterials Science. 2017; 5:1736-1745

[12] Abdolmaleki A, Ghasemi F, Ghasemi JB. Computer-aided drug design to explore cyclodextrin therapeutics and biomedical applications. Chemical Biology \& Drug Design. 2017; 89:257-268

[13] Alsbaiee A, Smith BJ, Xiao L, Ling Y, Helbling DE, Dichtel WR. Rapid removal of organic micropollutants from water by a porous $\beta$-cyclodextrin polymer. Nature. 2016;529:190-194 
[14] Peng L, Liu S, Feng A, Yuan J. Polymeric nanocarriers based on cyclodextrins for drug delivery: Host-guest interaction as stimuli responsive linker. Molecular Pharmaceutics. 2017;14:2475-2486

[15] Schmidt BVKJ, Barner-Kowollik C. Dynamic macromolecular material design - The versatility of cyclodextrin-based host-guest chemistry. Angewandte Chemie International Edition. 2017;56:8350-8369

[16] Harada A, Kobayashi R, Takashima Y, Hashidzume A, Yamaguchi H. Macroscopic selfassembly through molecular recognition. Nature Chemistry. 2011;3:34-37

[17] Medronho B, Andrade R, Vivod V, Ostlund A, Miguel MG, Lindman B, Voncina B, Valente AJM. Cyclodextrin-grafted cellulose: Physico-chemical characterization. Carbohydrate Polymers. 2013;93:324-330

[18] Krings JA, Vonhoren B, Tegeder P, Siozios V, Peterlechner M, Ravoo BJ. Light-responsive aggregation of [small beta]-cyclodextrin covered silica nanoparticles. Journal of Materials Chemistry A. 2014;2:9587-9593

[19] Polarz S, Smarsly B, Bronstein L, Antonietti M. From cyclodextrin assemblies to porous materials by silica Templating. Angewandte Chemie International Edition. 2001;40: $4417-4421$

[20] Loftsson T. Self-assembled cyclodextrin nanoparticles and drug delivery. Journal of Inclusion Phenomena and Macrocyclic Chemistry. 2014;80:1-7

[21] Messner M, Kurkov SV, Jansook P, Loftsson T. Self-assembled cyclodextrin aggregates and nanoparticles. International Journal of Pharmaceutics. 2010;387:199-208

[22] He Y, Fu P, Shen X, Gao H. Cyclodextrin-based aggregates and characterization by microscopy. Micron. 2008;39:495-516

[23] Lo Nostro P, Lopes JR, Ninham BW, Baglioni P. Effect of cations and anions on the formation of polypseudorotaxanes. The Journal of Physical Chemistry B. 2002;106:2166-2174

[24] Becheri A, Lo Nostro P, Ninham BW, Baglioni P. The curious world of polypseudorotaxanes: Cyclodextrins as probes of water structure. The Journal of Physical Chemistry B. 2003;107:3979-3987

[25] De Sousa FB, Lima AC, Denadai AML, Anconi CPA, De Almeida WB, Novato WTG, Dos Santos HF, Drum CL, Langer R, Sinisterra RD. Superstructure based on [small beta]-CD self-assembly induced by a small guest molecule. Physical Chemistry Chemical Physics. 2012;14:1934-1944

[26] Sabadini E, Cosgrove T, Egídio FdC. Solubility of cyclomaltooligosaccharides (cyclodextrins) in $\mathrm{H}_{2} \mathrm{O}$ and $\mathrm{D}_{2} \mathrm{O}$ : A comparative study. Carbohydrate Research. 2006;341:270-274

[27] Naidoo KJ, Chen JY-J, Jansson JLM, Widmalm G, Maliniak A. Molecular properties related to the anomalous solubility of $\beta$-cyclodextrin. The Journal of Physical Chemistry B. 2004;108:4236-4238 
[28] Cai W, Sun T, Shao X, Chipot C. Can the anomalous aqueous solubility of [small beta]cyclodextrin be explained by its hydration free energy alone? Physical Chemistry Chemical Physics. 2008;10:3236-3243

[29] González-Gaitano G, Rodríguez P, Isasi JR, Fuentes M, Tardajos G, Sánchez M. The aggregation of cyclodextrins as studied by photon correlation spectroscopy. Journal of Inclusion Phenomena and Macrocyclic Chemistry. 2002;44:101-105

[30] Chen G, Jiang M. Cyclodextrin-based inclusion complexation bridging supramolecular chemistry and macromolecular self-assembly. Chemical Society Reviews. 2011;40: 2254-2266

[31] Zaman H, Bright AG, Adams K, Goodall DM, Forbes RT. Characterisation of aggregates of cyclodextrin-drug complexes using Taylor dispersion analysis. International Journal of Pharmaceutics. 2017;522:98-109

[32] Stappaerts J, Do Thi T, Dominguez-Vega E, Somsen GW, Van den Mooter G, Augustijns $P$. The impact of guest compounds on cyclodextrin aggregation behavior: A series of structurally related parabens. International Journal of Pharmaceutics. 2017;529:442-450

[33] Szejtli J. Introduction and general overview of cyclodextrin chemistry. Chemical Reviews. 1998;98:1743-1754

[34] Raffaini G, Mazzaglia A, Ganazzoli F. Aggregation behaviour of amphiphilic cyclodextrins: The nucleation stage by atomistic molecular dynamics simulations. Beilstein Journal of Organic Chemistry. 2015;11:2459-2473

[35] Raffaini G, Ganazzoli F, Mazzaglia A. Aggregation behavior of amphiphilic cyclodextrins in a nonpolar solvent: Evidence of large-scale structures by atomistic molecular dynamics simulations and solution studies. Beilstein Journal of Organic Chemistry. 2016; 12:73-80

[36] Cova TFGG, Nunes SCC, Pais AACC. Free-energy patterns in inclusion complexes: The relevance of non-included moieties in the stability constants. Physical Chemistry Chemical Physics. 2017;19:5209-5221

[37] Mixcoha E, Campos-Terán J, Piñeiro Á. Surface adsorption and bulk aggregation of cyclodextrins by computational molecular dynamics simulations as a function of temperature: $\alpha$-CD vs $\beta$-CD. The Journal of Physical Chemistry B. 2014;118:6999-7011

[38] López CA, de Vries AH, Marrink SJ. Molecular mechanism of cyclodextrin mediated cholesterol extraction. PLoS Computational Biology. 2011;7:e1002020

[39] Zhu X, Wu G, Chen D. Molecular dynamics simulation of cyclodextrin aggregation and extraction of anthracene from non-aqueous liquid phase. Journal of Hazardous Materials. 2016;320:169-175

[40] Lucio D, Irache JM, Font M, Martínez-Ohárriz MC. Nanoaggregation of inclusion complexes of glibenclamide with cyclodextrins. International Journal of Pharmaceutics. 2017; 519:263-271 
[41] Ghosh P, Maity A, Das T, Dash J, Purkayastha P. Modulation of small molecule induced architecture of cyclodextrin aggregation by guest structure and host size. The Journal of Physical Chemistry C. 2011;115:20970-20977

[42] Koichiro M, Masahiro S, Masayuki N. Viscosity B-coefficients, apparent molar volumes, and activity coefficients for $\alpha$ - and $\gamma$-cyclodextrins in aqueous solutions. Bulletin of the Chemical Society of Japan. 1983;56:3556-3560

[43] Marcus Y. Effect of ions on the structure of water: Structure making and breaking. Chemical Reviews. 2009;109:1346-1370

[44] Häusler O, Müller-Goymann CC. Properties and structure of aqueous solutions of hydroxypropyl-beta-cyclodextrin. Starch-Stärke. 1993;45:183-187

[45] Szente L, Szejtli J, Kis GL. Spontaneous opalescence of aqueous $\gamma$-cyclodextrin solutions: Complex formation or self-aggregation? Journal of Pharmaceutical Sciences. 1998;87:778-781

[46] Coleman AW, Nicolis I, Keller N, Dalbiez JP. Aggregation of cyclodextrins: An explanation of the abnormal solubility of $\beta$-cyclodextrin. Journal of Inclusion Phenomena and Molecular Recognition in Chemistry. 1992;13:139-143

[47] Wu A, Shen X, He Y. Micrometer-sized rodlike structure formed by the secondary assembly of cyclodextrin nanotube. Journal of Colloid and Interface Science. 2006;302:87-94

[48] Wu A, Shen X, He Y. Investigation on $\gamma$-cyclodextrin nanotube induced by N,N'-diphenylbenzidine molecule. Journal of Colloid and Interface Science. 2006;297:525-533

[49] Puskás I, Schrott M, Malanga M, Szente L. Characterization and control of the aggregation behavior of cyclodextrins. Journal of Inclusion Phenomena and Macrocyclic Chemistry. 2013;75:269-276

[50] Bonini M, Rossi S, Karlsson G, Almgren M, Lo Nostro P, Baglioni P. Self-assembly of $\beta$-cyclodextrin in water. Part 1: Cryo-TEM and dynamic and static light scattering. Langmuir. 2006;22:1478-1484

[51] Rossi S, Bonini M, Lo Nostro P, Baglioni P. Self-assembly of $\beta$-cyclodextrin in water. 2. Electron spin resonance. Langmuir. 2007;23:10959-10967

[52] Rao JP, Geckeler KE. Cyclodextrin supramacromolecules: Unexpected formation in aqueous phase under ambient conditions. Macromolecular Rapid Communications. 2011; 32:426-430

[53] De M, You C-C, Srivastava S, Rotello VM. Biomimetic interactions of proteins with functionalized nanoparticles: A thermodynamic study. Journal of the American Chemical Society. 2007;129:10747-10753

[54] Saokham P, Loftsson T. A new approach for quantitative determination of $\gamma$-cyclodextrin in aqueous solutions: Application in aggregate determinations and solubility in hydrocortisone $/ \gamma$-cyclodextrin inclusion complex. Journal of Pharmaceutical Sciences. 2015; 104:3925-3933 
[55] Do TT, Van Hooghten R, Van den Mooter G. A study of the aggregation of cyclodextrins: Determination of the critical aggregation concentration, size of aggregates and thermodynamics using isodesmic and $\mathrm{K} 2-\mathrm{K}$ models. International Journal of Pharmaceutics. 2017;521:318-326

[56] Valente AJM, Carvalho RA, Söderman O. Do cyclodextrins aggregate in water? Insights from NMR experiments. Langmuir. 2015;31:6314-6320

[57] Valente AJM, Carvalho RA, Murtinho D, Söderman O. Molecular dynamics of cyclodextrins in water solutions from NMR deuterium relaxation: Implications for cyclodextrin aggregation. Langmuir. 2017;33:8233-8238

[58] Ribeiro ACF, Leaist DG, Esteso MA, Lobo VMM, Valente AJM, Santos CIAV, Cabral AMTDPV, Veiga FJB. Binary mutual diffusion coefficients of aqueous solutions of $\beta$-cyclodextrin at temperatures from 298.15 to $312.15 \mathrm{~K}$. Journal of Chemical \& Engineering Data. 2006;51:1368-1371

[59] Ribeiro ACF, Santos CIAV, Valente AJM, Ascenso OS, Lobo VMM, Burrows HD, Cabral AMTDPV, Veiga FJB, Teijeiro C, Esteso MA. Some transport properties of $\gamma$-cyclodextrin aqueous solutions at (298.15 and 310.15) K. Journal of Chemical \& Engineering Data. 2008;53:755-759

[60] Ribeiro ACF, Valente AJM, Santos CIAV, Prazeres PMRA, Lobo VMM, Burrows HD, Esteso MA, Cabral AMTDPV, Veiga FJB. Binary mutual diffusion coefficients of aqueous solutions of $\alpha$-cyclodextrin, 2-hydroxypropyl- $\alpha$-cyclodextrin, and 2-hydroxypropyl- $\beta$ cyclodextrin at temperatures from (298.15 to 312.15) K. Journal of Chemical \& Engineering Data. 2007;52:586-590

[61] He J, Chipot C, Shao X, Cai W. Cooperative recruitment of amphotericin B mediated by a cyclodextrin dimer. The Journal of Physical Chemistry C. 2014;118:24173-24180

[62] Figueiras A, Sarraguça JMG, Carvalho RA, Pais AACC, Veiga FJB. Interaction of omeprazole with a methylated derivative of $\beta$-cyclodextrin: Phase solubility, NMR spectroscopy and molecular simulation. Pharmaceutical Research. 2007;24:377-389

[63] Figueiras A, Nunes SCC, Simões S, Pais AACC, Veiga F. Molecular interaction governing solubility and release profiles in supramolecular systems containing fenbufen, pluronics and cyclodextrins. Journal of Inclusion Phenomena and Macrocyclic Chemistry. 2015;81:395-407

[64] Li K, Wu X, Gu Q, Zhao X, Yuan M, Ma W, Ni W, Hou Z. Inclusion complexes of organic salts with [small beta]-cyclodextrin as organocatalysts for $\mathrm{CO}_{2}$ cycloaddition with epoxides. RSC Advances. 2017;7:14721-14732

[65] Mele A, Mendichi R, Selva A. Non-covalent associations of cyclomaltooligosaccharides (cyclodextrins) with trans- $\beta$-carotene in water: Evidence for the formation of large aggregates by light scattering and NMR spectroscopy. Carbohydrate Research. 1998;310:261-267 
[66] Hernández R, Rusa M, Rusa CC, López D, Mijangos C, Tonelli AE. Controlling PVA hydrogels with $\gamma$-cyclodextrin. Macromolecules. 2004;37:9620-9625

[67] Rusa CC, Fox J, Tonelli AE. Competitive formation of polymer-cyclodextrin inclusion compounds. Macromolecules. 2003;36:2742-2747

[68] Rusa CC, Bullions TA, Fox J, Porbeni FE, Wang X, Tonelli AE. Inclusion compound formation with a new columnar cyclodextrin host. Langmuir. 2002;18:10016-10023

[69] Papancea A, Valente AJM, Patachia S, Miguel MG, Lindman B. PVA-DNA cryogel membranes: Characterization, swelling, and transport studies. Langmuir. 2008;24:273-279

[70] Zerkoune L, Angelova A, Lesieur S. Nano-assemblies of modified cyclodextrins and their complexes with guest molecules: Incorporation in nanostructured membranes and amphiphile nanoarchitectonics design. Nanomaterials. 2014;4:741

[71] Auzély-Velty R, Djedaïni-Pilard F, Désert S, Perly B, Zemb T. Micellization of hydrophobically modified cyclodextrins. 1. Micellar structure. Langmuir. 2000;16:3727-3734

[72] Wang T, Chipot C, Shao X, Cai W. Structural characterization of micelles formed of cholesteryl-functionalized cyclodextrins. Langmuir. 2011;27:91-97

[73] Sliwa W, Girek T. Cyclodextrins: Properties and Applications. Weinheim, Germany: John Wiley \& Sons; 2016

[74] Mazzaglia A, Bondì ML, Scala A, Zito F, Barbieri G, Crea F, Vianelli G, Mineo P, Fiore T, Pellerito C, Pellerito L, Costa MA. Supramolecular assemblies based on complexes of nonionic amphiphilic cyclodextrins and a meso-tetra(4-sulfonatophenyl)porphine tributyltin(IV) derivative: Potential nanotherapeutics against melanoma. Biomacromolecules. 2013;14:3820-3829

[75] Jiang B-P, Guo D-S, Liu Y. Self-assembly of amphiphilic perylene-cyclodextrin conjugate and vapor sensing for organic amines. The Journal of Organic Chemistry. 2010; 75:7258-7264

[76] Bauer M, Charitat T, Fajolles C, Fragneto G, Daillant J. Insertion properties of cholesteryl cyclodextrins in phospholipid membranes: A molecular study. Soft Matter. 2012; 8:942-953

[77] Zhang H, Tan T, Feng W, van der Spoel D. Molecular recognition in different environments: $\beta$-cyclodextrin dimer formation in organic solvents. The Journal of Physical Chemistry B. 2012;116:12684-12693

[78] Zhang H, Tan T, Hetényi C, Lv Y, van der Spoel D. Cooperative binding of cyclodextrin dimers to isoflavone analogues elucidated by free energy calculations. The Journal of Physical Chemistry C. 2014;118:7163-7173

[79] Zhang H, Tan T, Hetényi C, van der Spoel D. Quantification of solvent contribution to the stability of noncovalent complexes. Journal of Chemical Theory and Computation. 2013;9:4542-4551 
[80] Zhang H, Tan T, van der Spoel D. Generalized born and explicit solvent models for free energy calculations in organic solvents: Cyclodextrin dimerization. Journal of Chemical Theory and Computation. 2015;11:5103-5113

[81] van der Spoel D, van Maaren PJ, Caleman C. GROMACS molecule \& liquid database. Bioinformatics. 2012;28:752-753

[82] Vanquelef E, Simon S, Marquant G, Garcia E, Klimerak G, Delepine JC, Cieplak P, Dupradeau F-Y. R.E.D. Server: A web service for deriving RESP and ESP charges and building force field libraries for new molecules and molecular fragments. Nucleic Acids Research. 2011;39:W511-W517

[83] Wong-ekkabut J, Karttunen M. The good, the bad and the user in soft matter simulations. Biochimica et Biophysica Acta (BBA) - Biomembranes. 2016;1858:2529-2538

[84] Hess B, Bekker H, Berendsen HJC, Fraaije JGEM. LINCS: A linear constraint solver for molecular simulations. Journal of Computational Chemistry. 1997;18:1463-1472

[85] Daura X, Gademann K, Jaun B, Seebach D, van Gunsteren WF, Mark AE. Peptide folding: When simulation meets experiment. Angewandte Chemie International Edition. 1999;38:236-240

[86] Das S, Subuddhi U. Exploring poly(vinyl alcohol) hydrogels containing drug-cyclodextrin complexes as controlled drug delivery systems. Journal of Applied Polymer Science. 2014;131:40318(1-13)

[87] Zhou J, Wang Q, Lu H, Zhang Q, Lv P, Wei Q. Preparation and characterization of electrospun polyvinyl alcoholstyrylpyridinium/ $\beta$-cyclodextrin composite nanofibers: Release behavior and potential use for wound dressing. Fibers and Polymers. 2016;17:1835-1841

[88] Ouyang L, Zhu L, Ruan Y, Tang H. Preparation of a native [small beta]-cyclodextrin modified plasmonic hydrogel substrate and its use as a surface-enhanced Raman scattering scaffold for antibiotics identification. Journal of Materials Chemistry C. 2015;3:7575-7582

[89] Knaapila M, Stewart B, Costa T, Rogers SE, Pragana J, Fonseca SM, Valente AJM, Ramos ML, Murtinho D, Pereira JC, Mallavia R, Burrows HD. Incorporation of a cationic conjugated polyelectrolyte CPE within an aqueous poly(vinyl alcohol) sol. Macromolecules. 2016;49:9119-9131

[90] Cezard C, Trivelli X, Aubry F, Djedaini-Pilard F, Dupradeau F-Y. Molecular dynamics studies of native and substituted cyclodextrins in different media: 1 . Charge derivation and force field performances. Physical Chemistry Chemical Physics. 2011;13:15103-15121

[91] Sousa da Silva AW, Vranken WF. ACPYPE-AnteChamber PYthon parser interfacE. BMC Research Notes. 2012;5:367 
